\title{
Research of Augmented Reality based on Unity3D JiaHui $\mathrm{Du}^{1}{ }^{\mathrm{a}}$, PengKai $\mathrm{Xu}^{1 \text { a }}$, TingYu Hou ${ }^{1}$ a, YaDong You ${ }^{1 \text { a }}$, Fei Guo ${ }^{1 \mathrm{a}}$ ${ }^{1}$ Electronic information engineering, Beijing Institute of Fashion Technology, Beijing 100029, China aemail: 939734689@qq.com
}

Keywords: Unity3D; Augmented reality. Monitor - -based; Qualcomm SDK

\begin{abstract}
At present, the development of the game is more and more quickly, in order to meet the demand of mass on the reality of the game interface, this paper introduces the technology of augmented reality and Unity3D respective characteristics and specific content, and then the analysis of augmented reality based on Unity3D, through the application of different methods, increase understanding of augmented reality. This paper systematically expounds the research progress in the field, for Unity3D for at technology development to solve the problem of carried on the thorough analysis and discussion, look forward to the challenges faced by the technology and the development prospect of the future.
\end{abstract}

\section{Introduction}

With the rapid development of modern science and technology and technology, more and more new technology has been developing, like augmented reality technology is one of the hand, it is a kind of information the real world and virtual world information "seamless" integration of new technologies, is originally in the real world within a certain time space is very difficult to experience the entity information (visual information, sound, smell, touch, etc.), by the science and technology such as computer, simulation and then overlay, the virtual information applied to the real world, was perceived by the human senses, sensory experience so as to be beyond the reality.

Environment of the real and virtual objects in real-time overlay to the same picture or space exist at the same time, it is a kind of wide application of emerging technologies now. Today the life of people more and more good, also more and more entertainment way, games have become an indispensable part of People's Daily life, how to do the game interface is more realistic, based on the reality scene into virtual element, will become the mainstream trend of people develop games, this article introduces a kind of augmented reality technology using Unity3D development method of the game.

\section{Augmented reality technology}

Augmented reality, hereinafter referred to as AR, not only showed the information of the real world, and the virtual information displayed at the same time, the two complement each other, stack. In the visualization of augmented reality, the user use of helmet mounted display, the real world with multiple synthesis in computer graphics, you can see the real world around it.

Augmented reality includes multimedia, three-dimensional modeling, real-time video display and control, multi-sensor fusion, real-time tracking and registration, fusion, etc. New technology and new methods. Augmented reality provided in the general case, different from humans can perceive information. AR system has three prominent characteristics: (1) the real world and virtual world of information integration; (2) with real-time interactive; (3) is located in virtual object in the three-dimensional space to add $^{[1]}$.

A complete the augmented reality system is composed of a set of tightly coupling, real-time work coordination with relevant software system hardware components.

\section{Monitor-Based}

In AR implementation scheme based on a computer monitor, camera intake in the real world 
image input to the computer, and computer graphics system of virtual scene synthesis, and output to the screen display. Enhanced user from the screen to see the final scene images. It is simple, but it can't bring how many immersive user. Monitor - -based augmented reality system implementation scheme as shown in the figure below.

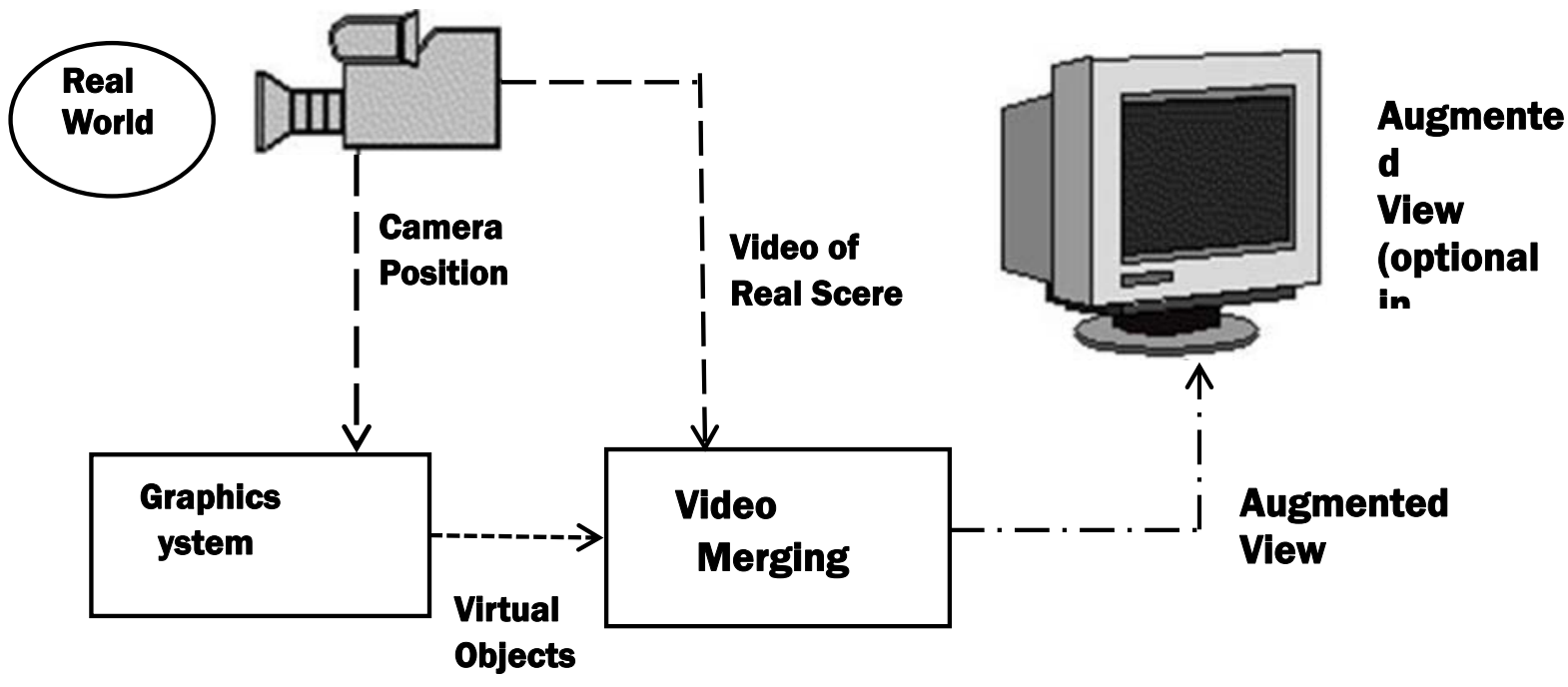

Monitor-Based augmented reality system

\section{Working principle:}

Below is for augmented reality system normal work the three components:

1. The head-mounted display.

2. Tracking system.

3. Mobile computing ability.

Augmented reality developer's goal is to integrate the three components into one unit, placed in binding with a girdle of equipment, the equipment can wirelessly relayed to the information is similar to ordinary glasses display.

\section{Introduction of Unity3D}

Unity3D is a cross-platform game engine, can carry out the development of PC and Mac OS, PS3, XBox360, Wii, iOS and Android platform game. Unity3D also can develop online games, Internet Explorer, Firefox, Google Chrome, Safari, install the plug-in can be executed after Unity3D game development. Unity3D provides easy-to-use interface operation, support PhysX physics engine, particle system, and provides the function of online multiplayer, do not need to learn complicated programming language, meet the various needs of game production. Released the latest version of Unity $3.5 \mathrm{x}$ provides the function of the Flash game. Unity3D lowered the threshold of the game development, and even personal studio games is no longer a dream. Using Unity3D for game companies can also shorten the development time of the game, and reduces the production cost of the game.

\section{Unity3D main functions}

Built-in NVIDIA PhysX physics engine to provide realistic physics calculations. Many Internet connections through RakNet support multiplayer online games at the same time. Graphical optimization have support Direct with OpenGL graphics optimization technology. Terrain processor make it easy for designers to quickly make natural terrain scene. 


\section{About augmented reality based on Unity3D}

Nowadays, there are a lot of games and applications of augmented reality on the market, first Unity3D development software is a very powerful development programs, and augmented reality is a very powerful technology, how to put the Unity3D game development software with augmented reality closely unifies in together, this is what I want to say next. Before they actually use Unity3D combined with augmented reality made some applications, such as Anatomy, $4 \mathrm{~d}$, with it, you can observe the human body structure of the complex anytime and anywhere, in a new way to study Anatomy, from the introduction to the application, developers can make the learning Anatomy or some people interested in human organs is able to more understanding to the human body, is this application can make use of the user's own photographs as the research object to study what you want to study human organs, Do you want to study the structure of the heart, for example, you can put a picture of you want to study heart import into this application, you can amplify the figure of the heart, and do all kinds of observation, research, for some people is a practical very strong application. In this method, I made a own idea, how the application of augmented reality to the Unity3D game, Unity3D general development is a kind of single machine game of the individual, we can use this technology, augmented reality of real-life scenarios to join in the game, to make the game more authenticity.

\section{Qualcomm SDK}

Qualcomm SDK is now the most comprehensive business functions augmented reality development kit, it by integrating the fit-out international patent technology, broad support iOS, Windows, PC and the android platform, but also to fully support the Unity $3 \mathrm{~d}$, is the most can represent the core technology of augmented reality software products. America qualcomm as pioneer and technology leader in the field of augmented reality, devoted to the interaction of digital virtual world and real world software products and technical solutions, make the $3 \mathrm{~d}$ virtual information and digital content seamlessly integrated into the computer and mobile terminal equipment real-time camera view, and published in multiple platforms.

Qualcomm has a diversified portfolio of products, ranging from mobile terminal to the desktop computer, from consumer applications to industrial, from hardware and software to optimize to the final product. Is different from the common GPS based augmented reality, augmented reality based on vision is qualcomm by optimizing the performance of the hardware and software, using the camera, processor and screen to identify the camera picture, with the computer to create virtual element integration, to provide a stronger sense of augmented reality experience.Specifically, qualcomm's augmented reality platform and software development kit (SDK) for the mobile terminal platform (iOS and Android) and PC platform provides the needed for creating interactive and immersive experience of augmented reality technology framework and function, mainly including the following: one is for iOS, Android, and Windows (PC) native application development; 2 it is built-in $3 \mathrm{~d}$ renderer mobile device can be a powerful $3 \mathrm{~d}$ tracking; Three is the Unity and development of plug-ins (only iOS and Android); Four is equipped with SLAM (simultaneous localization and mapping), but in the real world scenario, create a temporary augmented reality content; Four is the advanced augmented reality technology, such as deployment, continuous visual search based on cloud computing, etc.

How to make real and virtual, this is about to use a new technology of increasing the effect, make the virtual reality to realize interaction with us, first of all, the system interactive, the user can through interactive devices to interact directly with the virtual object or virtual environment.

Early set design - is the character, background, props. Before this belong to the previous Settings, including script, story board, mirror. Software can be used by any software drawing to do video.

According to the model, set up the character, background, props. Relatively the most difficult role model, because after wiring involved in binding and character animation module, is not independent, no art strength structure, it is difficult to be also to two or morethings wiring structure. General and simple model using Maya, $3 \mathrm{~d}$ Max, and so on mainstream software can complete, 
advanced model also can use these mainstream $3 \mathrm{~d}$ software, but involves the ultra-high precision of the model, is used specially to make $3 \mathrm{~d}$ model of the software.

Binding, according to the model group provides the model and collect the opinions of the character animation, binding level to produce products that meet the project requirement, can say without this module, there is no $3 \mathrm{~d}$ character animation, let him in accordance with the laws of physics to exercise. Are generally use mainstream software can own binding system, and some auxiliary plug-in.

Character animation - as the name implies, let all things without life, work, not includes nature of life and nature of life, anything can be a character animator "character". Posing as a model, save frame, then put the follow-up modelling, producers now just need to keep key frames, processing need to manually adjust the, most of the frames are computing, computing the trajectory can also control, 24 frames a second mostly, short shots are ok, 10 seconds of a frame of a frame of lens.

Middle add texture, lighting, special effects, etc. Later you can talk with realistic images of synthesis, this will make it so that we normally achieve the enhancement purpose to the effect of pictures.

\section{The main technical problems:}

Augmented Reality's key component is image identification, Image recognition algorithm refers to the use of texture, color characteristics to distinguish the target from the image, according to the characteristics of the use of different, divided into two categories, global and local features. Method of global by comparing to identify the images and the atlas inosculation template library to distinguish targets, using a statistical classification technique. Global method to target recognition of important issues, such as complicated block relations, illumination and zooming, is hard working, scope of application is limited. The local feature descriptor is using of objects' local feature such as key points or key edges to describe. From to identify the characteristics of the image feature set to the template image set of mapping process is called a matching. Able to correctly match the feature points are known as interior point, conversely for outside. As long as there is sufficient interior point, even if part of the feature points matching is less than, still can achieve effective identify and locate And the error matching can be avoided by effective strategies [2]. Because of the complexity of the augmented reality application scenario, the requirement of feature descriptor is not sensitive to visual Angle and the light changes. SIFT and SURF as described by the local feature descriptors, on the basis of reducing the amount of data can be a very good solve to the problem of shade and so on, of course become by far the most common and most popular image recognition algorithm. In order to meet their different needs, at present there are many improvements based on these two operators.

The angular point's identification and location is a corner detection method, the angular point is not clearly defined, it is generally believed angular point is a $2 \mathrm{~d}$ image brightness change sharply point or edge points on the curve which curvature is maximal. When these points keeping the features of the image, they effectively improving the calculation speed, reliable for image matching, that makes it possible to real-time processing.

\section{Moravec Corner detection algorithm}

Moravec corner detection algorithm is put forward by Moravec in $1977^{[3]}$, is the earliest one of the corner detection algorithms. The method of angular point is defined as in all directions (vertical, horizontal and diagonal) have intense intensity variations. When running, the moving of method in each direction is a testing point centered small rectangular window, through calculating before and after the sum of the squares of the two pixel gradient within the moment of windows to obtain the intensity variations in all directions. At last, the method selects the minimum value of eight directions' intensity variations as intensity variation in that point. If the value is greater than the threshold, then think that point as the angular point.

The calculation of intensity variation is as shown in figure 1-1. The corner threshold for 500, and 
the window size is $5 * 5$, Moravec corner detection algorithm's results are shown in figure 1-2. Moravec corner detection algorithm is relatively simple, calculating speed is fast, and meeting the real time, but because of there were only eight directions' intensity variations are calculated, so it doesn't have rotation invariance. At the same time, due to the calculation of gradient with the rectangular window, relative to the circular windows are more likely to introduce calculation error, and at the meanwhile the result is very sensitive to strong edge, thus the result obtains many corners in error. With the strengthening of computers' computing ability, the method has been used less.

\begin{tabular}{|l|l|l|l|l|l|}
\hline & & & & & \\
\hline & & B1 & B2 & B3 & \\
\hline & A1 & A2 & A3 & B6 & \\
& & B4 & B5 & & \\
\hline & A4 & A5 & A6 & B6 & \\
& A6 & B8 & & \\
\hline & A7 & A8 & A9 & & \\
\hline & & & & & \\
& & & & & \\
\hline
\end{tabular}

$\mathrm{V}=\sum_{i=1}^{9}\left(A_{i}-B_{i}\right)-2 * 255^{2}$

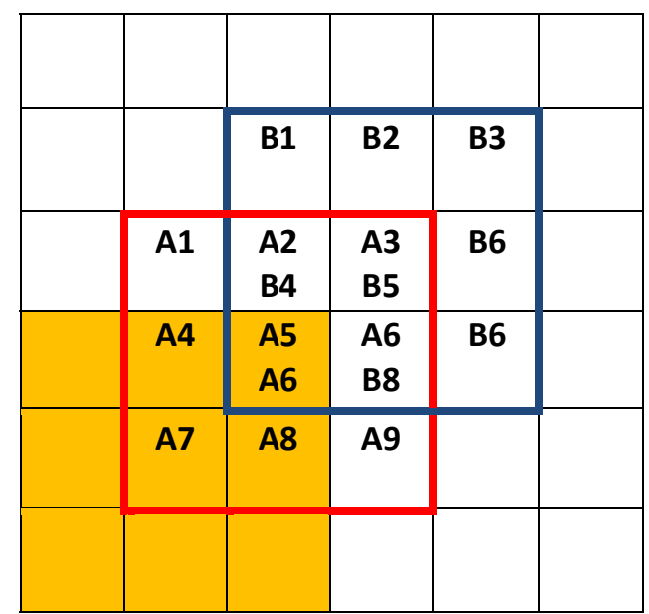

$$
V=\sum_{i=1}^{9}\left(A_{i}-B_{i}\right)-3 * 255^{2}
$$

Fig. 1-1 Calculations of intensity variation for Moravec operator

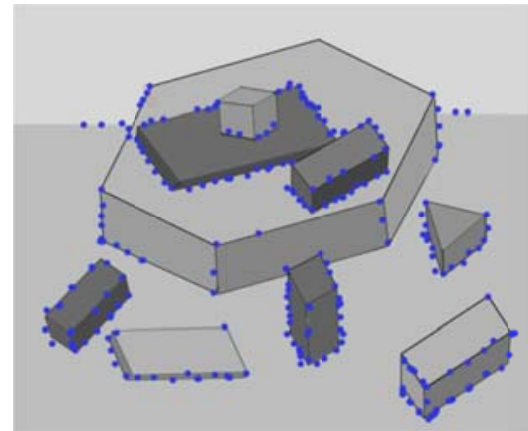

Fig. 1-2 Result of the Moravec operator

\section{Harris Corner detection algorithm}

Harris corner detection algorithm is also called Plessey corner detection algorithm, put forward by Harris and Stephens in $1988^{[4]}$. This algorithm inspired by autocorrelation function in signal processing, and gives the correlation matrix $\mathrm{M}$ corresponding to the autocorrelation function. The characteristic value of $\mathrm{M}$ matrix is the first order curvature of the autocorrelation function. If the two curvature values are high, it is considered that the point is the characteristic point. The basic idea of this method is similar to the Moravec corner detection algorithm, but the disadvantage of Moravec corner detection algorithm is improved. Firstly, the Harris corner detection algorithm using Taylor expansion to approximate arbitrary direction to the anisotropic problem in Moravec algorithm. Secondly, the Harris corner detection algorithm is used to avoid the effect of noise using the Gaussian smoothing window. Finally, a new corner detection algorithm based on Harris corner detection is used to eliminate the sensitivity of Moravec algorithm to the strong edge. Therefore, the Harris corner detection algorithm is a kind of stable and effective corner detection algorithm, and is currently the most widely used corner detection algorithm.

Harris corner detection algorithm of the specific steps is as follows: 
Firstly, the calculation of each pixel correlation matrix $M$ to describe the gradient distribution of pixels in the neighborhood of each pixel:

$$
\mathrm{M}=\sigma_{D}^{2} \mathrm{~g}\left(\sigma_{1}\right) *\left[\begin{array}{cc}
I_{x}^{2}\left(x, \sigma_{D}\right) & I_{x}\left(x, \sigma_{D}\right) I_{y}\left(x, \sigma_{D}\right) \\
I_{x}\left(x, \sigma_{D}\right) & I_{y}\left(x, \sigma_{D}\right) I_{y}^{2}\left(x, \sigma_{D}\right)
\end{array}\right]
$$

There into

$$
\begin{aligned}
& I_{x}\left(x, \sigma_{D}\right)=\frac{\partial}{\partial x} \mathrm{~g}\left(\sigma_{D}\right) * I(x) \\
& \mathrm{g}(\partial)=\frac{1}{2 \pi \sigma^{2}} \exp \left(-\frac{x^{2}+y^{2}}{2 \partial^{2}}\right)
\end{aligned}
$$

Then, the Harris corner response value of each pixel is calculated:

\section{Cornerness $=\operatorname{det}(M)-\lambda \operatorname{trace}(M)$}

Type in the $\lambda$ value generally take 0.4 . If the angular point response vallue is greater than the threshold, it is considered that the point is the corner point. When the response threshold is 500, Harris corner detection algorithm is shown in figure 1-3. Harris corner detection algorithm is an effective corner detection algorithm, this method has the following characteristics: (1) Simple calculation, the Harris operator only uses the first order difference of gray scale and filtering, the operation is simple. (2) The extracted corner uniform and reasonable, Harris operator is used to calculate the value of interest for each point in the image, and then select the most merit in the neighborhood. (3) The stability, the calculation formula of the Harris operator is only related to the first derivative, therefore, the image rotation, gray level changes and the impact of noise are not sensitive, but the Harris corner detection algorithm is sensitive to the scale, generally do not apply to the existence of scale changes.

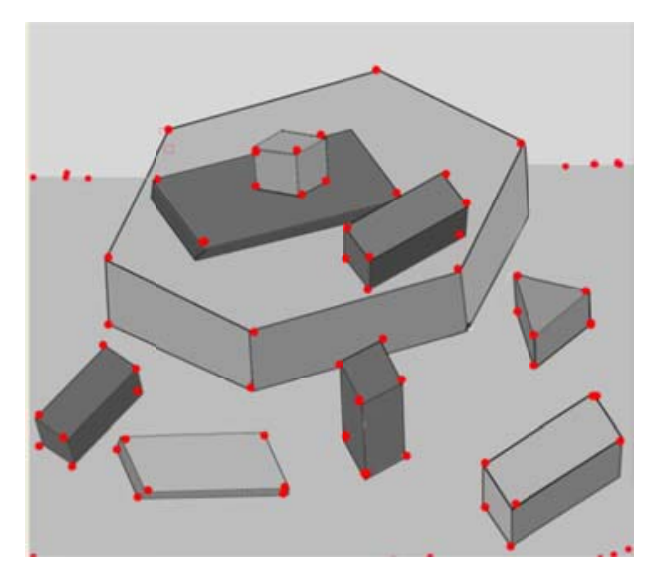

Fig. 1-3 Result of the Harris corner detector

\section{SUSAN corner detection algorithm}

SUSAN corner detection algorithm is proposed by Smith and Brady ${ }^{[5]}$ in 1997, which is called Small-est Univalue Segment Assimilating Nucleus, meaning assimilation nuclear division minimum value. The algorithm firstly establishes a circular mask, saying its mask center as the core of the mask. Move the mask on the image to be detected, if the mask covering the grey value of points in the area is similar to mask the nuclear of grey value, says these points for the area is USAN (Univalue Segment Assimilating Nucleus), namely the assimilation of nuclear division area of the same value. The USAN region contains important information about the image information. The USAN value is close to half of the maximum value when the mask is near the edge of the image, and the USAN value is close to $1 / 4$ of the maximum value when the mask is in a corner of the image. Therefore, the detection of the corner is converted into a mask that is less than a certain threshold value for detecting the USAN value. 
SUSAN corner detection procedures ${ }^{[04]}$ are as follows:

First, a circular mask is constructed, and the USAN values are calculated at each point of the image, and the USAN values are calculated using the following functions:

$$
U S A N=\sum C(x, y)
$$

$\mathrm{C}(\mathrm{x}, \mathrm{y})$ is the similarity between the pixels in the mask and the mask core, which is calculated as follows:

$$
C(x, y)=\exp \left(-\left(\frac{I(x, y)-I\left(x_{0}, y_{0}\right)}{t}\right)^{6}\right)
$$

Among them, (x0, Y0) is the coordinates of the mask nuclear in the image, $(\mathrm{x}, \mathrm{y})$ for the coordinates of the other points in the mask region. I (x, y), I (x0, y 0$)$ respectively point $(\mathrm{x}, \mathrm{y})$ and $(\mathrm{x} 0, \mathrm{y} 0)$ grey value. The threshold $\mathrm{t}$ determines the maximum difference in similar of two points.

After obtaining the USAN value, compare it with a specific threshold, if it is less than a certain threshold, it is considered as the corner point When the mask size is 37 , the SUSAN corner detection algorithm is shown in Figure 1-4.

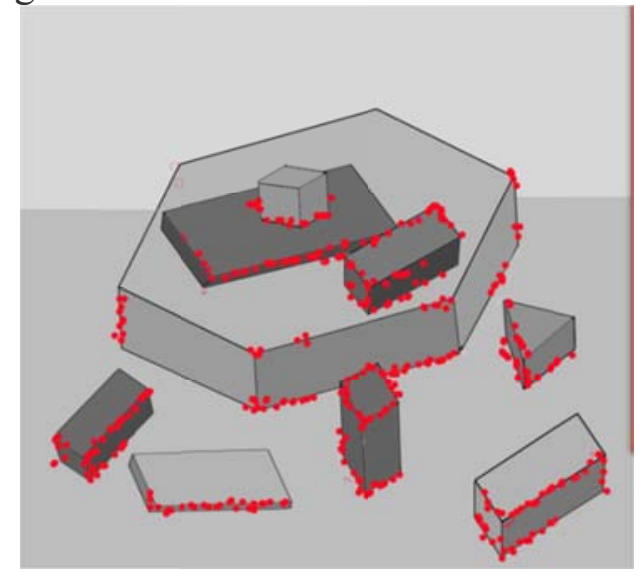

Fig. 1-4 Result of the SUSAN corner detector

SUSAN corner detection algorithm is also a good point detection algorithm. This method can be used to locate the corner point accurately, but also has the ability of anti -noise, so it has been widely used. However, this method has poor detection effect on fuzzy images, which also limits its application scope.

\section{Conclusion}

From the above analysis, augmented reality will play a very important role in our life, and we will use Unity3D in augmented reality, it can be easier to achieve augmented reality becomes possible, so that we will feel more than the reality of the experience. Now the main problem is the image recognition, we use the corner detection method is very effective to solve this problem. Research on augmented reality will show more achievements in the future, we will have more new technology to solve the related problems, augmented reality will bring us more experience.

\section{Acknowledgement}

In this paper, the research was sponsored by the General Program of Science and Technology Development Project of Beijing Municipal Commission of Education (Project No. KM201410012001) and National Science and Technology Supporting Funding Project(Project No. 2013BAH41F02) and Youth Innovation Fund Project of science of Beijing Institute Of Fashion Technology (2015AL-29) and 2015 College Student Research Training Program of Beijing Institute Of Fashion Technology and General program of science and technology development project of Beijing Municipal Education Commission (KM201510012005) and Beijing Institute of Fashion Technology Teaching reform and innovation team project ( JGTD-1404) and Funding Project for 
Academic Human Resources Development in Institutions of Higher Learning Under the Jurisdiction of Beijing Municipality, PHR(IHLB) of Innovative Research Team PTTBIFT_TD_002 and Project of Beijing Institute of Fashion Technology Specialty construction(RCPY20150024). Teacher Fei Guo is corresponding author.

\section{References}

[1] Ronald T. Azuma. A Survey of augmented reality [J]. Teleoperators and VirtualEnvironments, 1997.6 (4) :355-385.

[2]Qiong Jia,Mingmin Wang.The augmented reality system based on object recognition [J].Microcomputer Applications,2013,6:006.

[3] H. Moravec, Towards Automatic Visual Obstacle Avoidance, Proceedings of International Conference .on Artificial Intelligence, 1977, 584.

[4] C. Harris and M. Stephens, A Combined Corner and Edge Detector, Alvey Vision Conference, $1988,147-151$.

[5] S. M. Smith and J. M. Brady, SUSAN - A New Approach to Low Level Image Processing,International Journal of Computer Vision, 1997, 23: 45-78. 